\title{
Reviewers for Volume 42
}

Teresa Abada, University of Western Ontario, London (Canada)

Akbar Aghajanian, Fayetteville State University NC (USA)

Ather Akbari, Saint Mary's University, Halifax (Canada)

Gunnar Andersson, Stockholm University (Sweden)

Sulaiman Bah, Sciences University of Dammam (Saudi Arabia)

Roderic Beaujot, University of Western Ontario, London (Canada)

Robert Bourbeau, Université de Montréal (Canada)

Emir Erfani, Nipissing University ON (Canada)

Janet Fast, University of Alberta, Edmonton (Canada)

Rejulton Fernando, University of Western Ontario, London (Canada)

Eric Fong, University of Toronto (Canada)

Nora (Bonhert) Galbraith, Statistics Canada, Ottawa (Canada)

Gustave Goldmann, Univeristé d'Ottawa (Canada)

Rafael Gomez, University of Toronto (Canada)

Benjamin Harris, U.S. Census Bureau, Washington (USA)

Homa Hoodfar, Concordia University, Québec (Canada)

Feng Hou, Statistics Canada, Ottawa (Canada)

Nora Keating, University of Alberta, Edmonton (Canada)

Don Kerr, University of Western Ontario, London (Canada)

Byron Kotzamanis, University of Thessaly, Volos (Greece)

Taki Kukutai, University of Waikato, Hamilton (New Zealand)

Rachel Margolis, University of Western Ontario, London (Canada)

Paul Maxim, Wilfrid Laurier University, Waterloo ON (Canada)

Ryan Mazan, Nunavut Bureau of Statistics, Iqaluit (Canada)

Ted McDonald, University of Toronto (Canada)

Javier Moguerza, Rey Juan Carlos University, Madrid (Spain)

Richard Mueller, University of Lethbridge AB (Canada)

Herb Northcott, University of Alberta, Edmonton (Canada)

Robert M. O’Brien, University of Oregon, Eugene (USA)

Mario Polèse, INRS Centre - Urbanisation Culture Société, Québec (Canada) 
Amelie Quesnel-Valle, McGill University, Montreal (Canada)

K.V. Rao, India Network Foundation, Orlando FL (USA)

Zeneida Ravanera, University of Western Ontario, London (Canada)

Anatole Romaniuc, University of Alberta, Edmonton (Canada)

Mark W. Rosenberg, Queen's University, Kingston ON (Canada)

Grant Schellenberg, Statistics Canada, Ottawa (Canada)

Alan Simmons, York University, Toronto (Canada)

Martin Spielauer, Statistics Canada, Ottawa (Canada)

Thomas Spoorenberg, Statistics Division, United Nations, New York (USA)

Steven Stack, Wayne State University, Detroit (USA)

Gillian Stevens, University of Alberta, Edmonton (Canada)

Chirayath M. Suchindran, University of North Carolina, Lafayette (USA)

Zongli Tang, University of Alabama at Montgomery (USA)

Jeff Tayman, University of California, San Diego (USA)

Lise Thibodeau, University of Montreal, Ottawa (Canada)

Mark Tolts, Hebrew University of Jerusalem, Mt. Scopus (Israel)

Rosemary Venne, University of Saskatchewan, Saskatoon (Canada)

Ravi Verma, Carleton University, Ottawa (Canada)

Barb Whittington, University of Victoria (Canada)

Zheng Wu, University of Victoria (Canada)

Ling Yang, Queen's Park, Toronto (Canada)

Yan Yaun, University of Alberta, Edmonton (Canada) 